\title{
Protective Role of Eurycoma longifolia Jack Root Extract Against High-Fat Diet Induced Testicular Damage in Sprague-Dawley Rats
}

\author{
Imad M Al-Ani' ${ }^{1}$, Norsidah Ku-Zaifah², Fakhria A. Al-Joufi ${ }^{3}$, Rafidah H.Mokhtar' ${ }^{4}$, Norlelawati A. Talib ${ }^{5}$, Ghasak \\ Ghazi Faisal6,*
}

Imad M Al-Ani ${ }^{1}$, Norsidah Ku-Zaifah², Fakhria A. AlJoufi ${ }^{3}$, Rafidah H.Mokhtar ${ }^{4}$, Norlelawati A. Talib ${ }^{5}$, Ghasak Ghazi Faisal ${ }^{6, *}$

'Department of Dentistry, Al-Hikmah University College, Al-Yarmook, Baghdad, IRAQ.

${ }^{2}$ Department of Basic Medical Sciences, Kulliyyah of Medicine, International Islamic University, MALAYSIA.

${ }^{3}$ College of Pharmacy, Al Jouf University, Aljouf, Skaka KSA.

4Faculty of Medicine, Universiti Sains Islam Malaysia, Nilai, Negeri Sembilan, MALAYSIA.

${ }^{5}$ Department of Pathology and Laboratory Medicine, Kulliyyah of Medicine, International Islamic University, MALAYSIA. ${ }^{6}$ Department of Fundamental Dental and Medical sciences, Kulliyyah of Dentistry,

International Islamic University, MALAYSIA.

\section{Correspondence}

Ghasak Ghazi Faisal

Department of Fundamental Dental and Medical sciences, Kulliyyah of

Dentistry, International Islamic University, MALAYSIA.

Tel. +0060105442293

E-mail: drghassak@yahoo.com

History

- Submission Date: 12-03-2019;

- Review completed: 15-04-2019;

- Accepted Date: 26-04-2019.

\section{DOI : 10.5530/pj.2019.11.129}

Article Available online

http://www.phcogj.com/v11/i4

Copyright

(C) 2019 Phcogj.Com. This is an openaccess article distributed under the terms of the Creative Commons Attribution 4.0 International license.

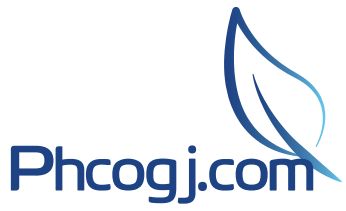

\begin{abstract}
Objective: The aims of this study were to investigate the adverse effects of the high-fat-diet (HFD) on the testosterone level and testicular structure of male rats as well as to examine whether Eurycoma Longifolia (EL) is able to ameliorate these effects. Methods: Twenty-four male Sprague-Dawley (SD) rats were divided into four groups each containing 6 rats. Group ND was given only normal diet, group NDEL was given normal diet and EL extracts (15 mg/ $\mathrm{kg}$ ) dissolved in distilled water, group HFD was given only high-fat-diet and group HFDEL was given high-fat-diet and EL extracts $(15 \mathrm{mg} / \mathrm{kg})$. EL was administered orally for 12 weeks. The animal's testosterone level was measured at week 0,6 and 12. The rats were sacrificed at the end of $12^{\text {th }}$ weeks and the testes samples were processed for histological examination Results: The testosterone level was significantly increased $(p<0.05)$ in the in the treated rats with EL (NDEL and HFDEL) compared with ND and HFD groups. Treatment with HFD revealed a marked degeneration of the seminiferous tubule epithelium and disruption of interstitial cells of the testis thereby interfering with spermatogenesis. Treatment of HFD rats with El reduced the adverse effects of HFD and improved the morphological structure of the seminiferous tubules. Conclusion: These findings revealed that EL has ameliorative effects against the testicular damage caused by high-fat-diet.
\end{abstract}

Key words: Eurycoma longifolia, High-fat-diet, Seminiferous tubules Tongkat Ali, Testosterone, Testis.

\section{INTRODUCTION}

Obesity, which can be due to high dietary fat intake, is a rapidly growing problem worldwide. ${ }^{1}$ It is designated as a circumstance of excessive and unusual fat aggregation that may unfavorably affect the health. More than 1.9 billion adults were registered overweight in 2016; and 650 million of them were obese, with 2.8 million persons pass away each year as a consequence of being overweight or obese. ${ }^{2}$ Obesity, is accompanied with diversity of chronic diseases, such as diabetes mellitus, hypertension, and hyperlipidemia. In addition, obesity, has also been linked to dangerous effects on spermatogenesis, which can affect the fertilization rate. ${ }^{3,4}$ Testosterone, the primary male sex hormone produced by Leydig cells, it plays a significant role in the development of male reproductive tissues and spermatogenesis.

Recently, many studies have used high-fat diet (HFD) to induce obesity by a nutritional involvement in several experimental animals, HFD has been shown to induce hyperglycemia and whole-body insulin resistance, many investigators have demonstrated that HFD induces lipid metabolic disorder and leads to a lack of male reproductive function, such as decreased serum testosterone level, sexual hormones metabolic disorder, apoptosis of seminiferous tubules cell and a significant deterioration of sperm function parameters, including a decrease in semen volume reduced score for sperm count, decreased motility associated with a significant increase in sperm cell malformation, and disturbing of blood testeis barier integrity. ${ }^{5-7}$

Recent studies using many herbal medicines exert protective and therapeutic effects on the testes and spermatogenesis in animals fed HFD. Curcumin has been shown to reduce HFD-induced spermatogenesis impairment and apoptosis of the seminiferous tubules. ${ }^{8}$ Nigella sativa enhance fertility by means of increasing the healthy sperm number and preventing sperm anomalies in HFD fed rats. ${ }^{9}$ Cinnamon significantly reduced the cholesterol, triglycerides, and low-density lipoprotein levels and increased high-density lipoprotein in rats fed with a high cholesterol diet "HCD" and improved the disorganized and shrinking of the seminiferous tubules of those received only the HCD. ${ }^{10}$

Eurycoma longifolia "EL" (publically known as Tongkat Ali) is a herbal medicinal plant found in South-East Asia, It is a small rain forest flowering, un-branched, medium-sized slender shrub. The root of the plant has been commonly consumed in traditional medicine for the treatment of many diseases in South-East Asia countries. EL has potent antibacterial, antimalarial, antipyretic, antifungal, and antioxidant properties. ${ }^{11,12}$

The roots of the EL contain numerous phytochemical bioactive compounds including quassinoids and squalene derivatives and eurycolactones A-C. ${ }^{13}$ 
The EL extract has exclusively been used for enhancing testosterone levels in males. ${ }^{14}$ It has theability to increase testosterone levels in the circulation of hypogonadic males and laboratory animals. ${ }^{15}$

In previous studies, the authors explored the consequences of EL treatment on atherosclerosis-related parameters in HFD fed rats; EL significantly reduced triglyceride elevated by HFD and attenuated atherosclerotic plaque developed in the aorta and maintain the normal vascular structure. ${ }^{16}$ In the current study we examined the protective effects of oral administration of EL on testosterone level and the histopathological changes in the testes of HFD fed rats.

\section{METHODOLOGY}

\section{Eurycoma longifolia root (EL) extract}

The root extract was purchased from Biotropics Malaysia. It was prepared and administered by oral gavage as described previously. ${ }^{17}$

\section{High-fat diet}

The high fat diet Pellets were purchased from MP Biomedicals, California, USA (Next Gene Scientific Sdn. Bhd).

\section{Animals and experimental design}

Prior to commencement of the study, an ethical approval was obtained for use of animals from the International Islamic University Animal use and care committee according to the Malaysia animal welfare act 2015 with reference number (IIUM/519/14/4/IACUC).

Twenty four healthy adult male SD rats weighing 250-300 g were kept under appropriate environmental conditions such as room temperature weeks as described in Table 1.

They were maintained at room temperature $\left(22-24^{\circ} \mathrm{C}\right)$ with adequate ventilation, $12 \mathrm{~h}$ light-dark cycle and about $(50 \pm 5 \%)$ humidity. After one week of acclimatization, they were randomly divided into four groups of 6 animals each and treated for 12 weeks as follow: Group ND was given only normal diet, group NDEL was given normal diet and EL extracts $(15 \mathrm{mg} / \mathrm{kg})$ dissolved in distilled water, group HFD was given only high fat diet and group HFDEL was given high fat diet and EL extracts $(15 \mathrm{mg} / \mathrm{kg})$.The EL extract was administered by gastric gavage.

At the end of $12^{\text {th }}$ week, the rats were kept in fasted state for 12 hours prior to anaesthesia and then sacrificed by cervical dislocation.

\section{Collection of blood samples}

Blood samples were obtained at week 0, 6 and at week 12. Blood samples were collected from all experimental groups under general anesthesia; the diethyl ether. A capillary tube was gently inserted in the optical sinus and the required volume of blood was collected in a sterile glass tube containing gel for serum separation. After allowing the blood to clot at room temperature for 20-30 minutes the blood samples were centrifuged at $3000 \mathrm{rpm}$ for 15 minutes. The centrifuged serum was stored at $-80^{\circ} \mathrm{C}$ untilthe biochemical evaluation was done.

\section{Testosterone assay}

Testosterone levels were directly assessed by chemiluminescent immunoassay (CLIA) on the high-performance ADVIA Centaur ${ }^{\circledR} \mathrm{XP}$ system as described previously. ${ }^{16}$

\section{Tissue specimens}

The rats were sacrificed at the end of $12^{\text {th }}$ weeks by cervical dislocation. The testis specimens were processed. Multiple $4 \mu \mathrm{m}$ sections were obtained and stained using haematoxylin and eosin (H\&E).

\section{Statistical analysis}

All analyses were conducted using one-way and repeated measures ANOVA using SPSS ver.22.0 software. To determine the significant difference among groups, Post-hoc comparison (Tukey's test) was used. Pearson's correlation coefficient was applied to calculate the correlation between total testosterone levels. $p<0.05$ was considered as statistically significant.

\section{RESULTS}

\section{Testosterone level}

The results of the present study revealed that there was no significant difference in the testosterone serum levels for all the groups at the beginning of study; while a significant elevation in testosterone level was observed during the treatment with EL at week 6, in both treated groups "NDEL and HFDEL" ( $p<0.05)$ compared with ND and HFD groups (ND vs. NDEL, $\mathrm{p}<0.05$ ) and (HFD vs. HFDEL, $\mathrm{p}<0.05)$. The F value was statistically significant $(\mathrm{F}(3,20)=52.3, \mathrm{p}<0.001))$, partial $\eta 2=0.89$, indicating that there was a difference of testosterone levels among groups over time. The rising tendency in both treated groups persisted till week 12 . The mean testosterone level of the NDEL group elevated from $3.017(0.36)(\mathrm{nmol} / \mathrm{l})$ to $8.3(1.2)(\mathrm{nmol} / \mathrm{l})$, moreover the mean testosterone level of HFDEL group increased from 3.87 (0.62) $(\mathrm{nmol} / \mathrm{l})$ to $8.13(1.2)(\mathrm{nmol} / \mathrm{l})$. While the mean of testosterone levels in ND and HFD were nearly identical throughout the duration of the experiment.

\section{Histopathological investigation}

Microscopic examination of testes sections from the rats of ND group "received normal diet" revealed normal seminiferous tubules (ST) architecture separated by interstitial connective tissue. The ST composed of regular cells of spermatogenic series orderly organized in the ST wall (Figures 1A and 1B). EL group rats "received normal diet and EL" exhibited normal morphological structure of ST, spermatogenic cells, and interstitial connective tissue with no indication of histological deformities (Figures 1C and 1D).

HFD (group HFD) leads to testicular damage, showing change in the general structure and shape of the ST, some tubules revealed disorganized and vacuolated germinal epithelium germinal epithelium, and thickness of the tubular walls was increased (Figures $1 \mathrm{E}$ and $1 \mathrm{~F}$ ).

HFD treated rats also showed congestion of blood vessels, degenerate parts of some seminiferous tubules, vacuolation of germinal epithelium with the absence of spermatogonial series and pyknotic nuclei of primary spermatocytes (Figures 2A-2D).

The rats of group HFDEL that were treated with HFD and EL showed that the ST acquired improvement in seminiferous tubules structure with normal complete spermatogenic series compared with HFD group as evident by the presence of normal testis architecture with regulated with normal STs diameter and absence of cellular damage (Figures $2 \mathrm{E}$ and $2 \mathrm{~F})$.

Table 1: Distribution of animals by groups.

\begin{tabular}{ccc}
\hline Group name & Group definition & Treatment \\
\hline ND & Normal diet control rats & Pellets and water \\
NDEL & Normal diet treated with EL & Pellets and water and 15 mglkg EL extract \\
HFD & HFD treated rats & High fat diet feeding \\
HFDEL & HFD and EL treated & High fat diet and EL \\
\hline
\end{tabular}



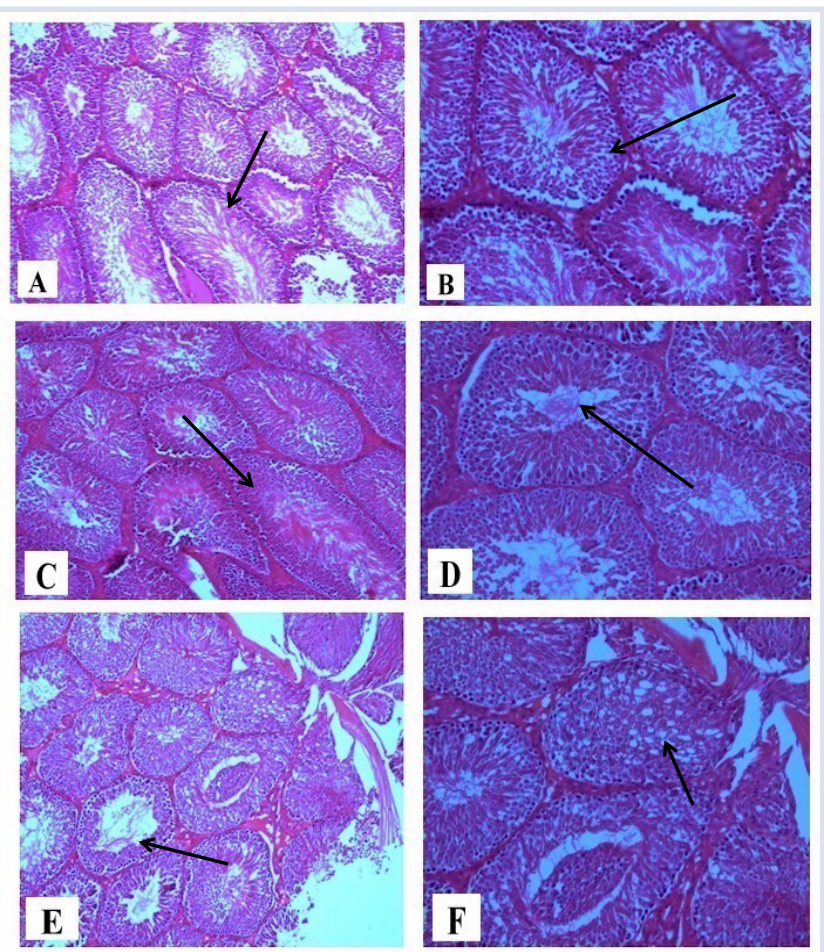

Figure 1: $(A, B)$ Control (ND) rat showing normal and regular seminiferous tubules (ST) architecture; (C, D) NDEL treated rat showing no evidence of histological abnormalities, they showed normal spermatogenesis in the $\mathrm{ST}$; $(\mathrm{E}, \mathrm{F})$ HFD treated rat; the seminiferous tubules displayed atrophy and vacuolation of germinal epithelium. (H\&E. A, C, and F; X100. B, D and F; X 200).
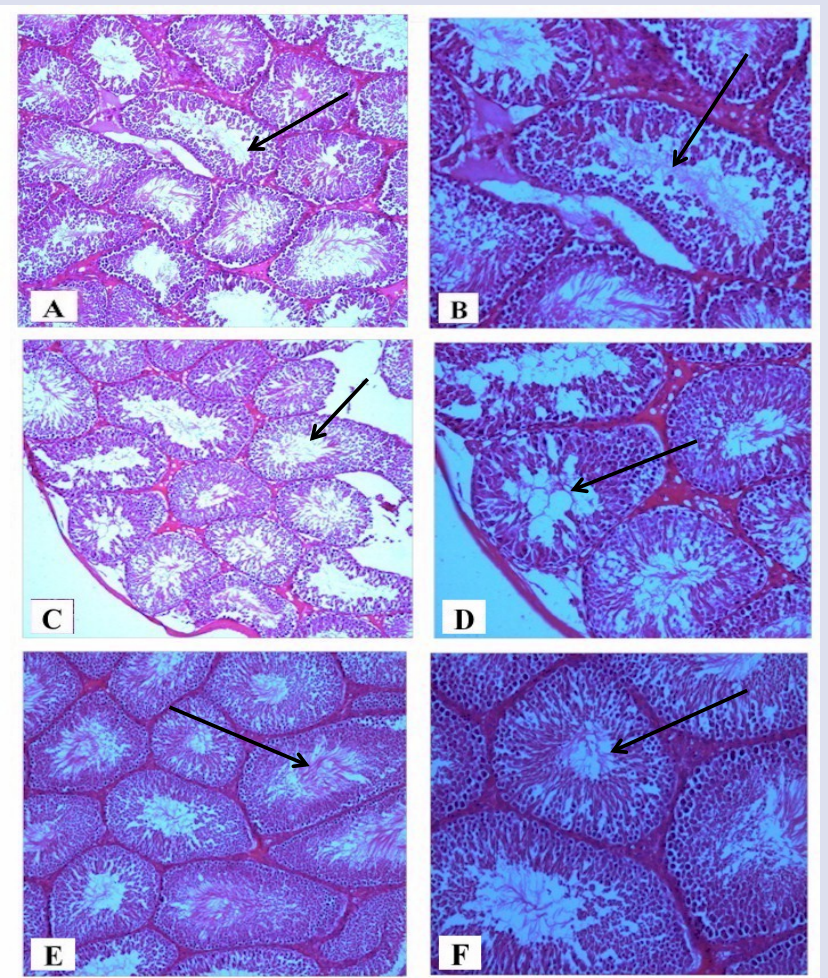

Figure 2: (A,B) HFD treated rat; the seminiferous tubules displayed atrophy and vacuolation and deposition of eosinophilic homogenous material in the interstitial tissue; $(C, D)$ HFD treated rat showing degeneration in most of the seminiferous tubules with the absence of spermatogonial series; $(E, F)$ HFDEL treated rat acquired improvement as evident by the presence of normal testis architecture and absence of cellular damage. (H\&E. A, C, and F; X100. B, D and F; X 200).

\section{DISCUSSION}

It has been shown that HFD altered the carbohydrate metabolism, the testicular architecture and leads to spermatogenetic damage and impaired the fertility. ${ }^{6,18,19}$ In the present study, serum testosterone, was comparable between normal diet and HFD animals, this in consent with Fernandez et al. who observed analogous testosterone levels in HFD rats compared with standard chow fed rats. ${ }^{20}$ Serum testosterone depletion accompanied with a reduction of sexual organ weight was observed in rats that fed on hypercaloric diet. ${ }^{21}$

The present histopathological result of HFD feeding is comparable to earlier studies of HFD-induced testicular injury; the HFD fed rats' revealed degeneration in testicular morphology, characterized as atrophied seminiferous tubules, disruption in spermatogenesis and vacuolation of germinal epithelium, this is contestant with previous studies who demonstrated atrophied seminiferous epithelia, coalescence between spermatogenic cells and impaired and loosely organized Sertoli cells in mice fed HFD. ${ }^{22}$ Atrophied seminiferous tubuli were also observed in HFD treated rats associated disconnection of the degenerated STs from the interstitial wall with increased area of the connective tissue and remarkable apoptotic activity in Leydig and Sertoli cells. ${ }^{9}$ Vacuolar alterations in seminiferous tubules, spermatogenic cell dysfunction, and increased apoptosis of spermatogenic cells in testicular tissue was also observed in HFD treated rats. ${ }^{23}$

Currently, many herbal medicines therapeutic available been recommended for the treatment of obesity. Eurycoma longifolia Jack (EL) or Tongkat Ali is a very well-known traditional medicine, EL has gained remarkable consideration in latest years because of its beneficial effects for many disorders and diseases and its strong sexual enhancing effects. ${ }^{15} \mathrm{EL}$, significant retrieve serum testosterone level further closely associated with medical circumstances such as obesity, metabolic syndrome, diabetes or hypertension, improving sexual health and demonstrated significant positive effects on bone health and physical condition of patients. ${ }^{24}$

After six weeks of treatment with EL (NDEL and HFDEL), the rats in the present investigation showed a significant elevation of testosterone levels when compared with the control groups (ND and HFD). This was supported by earlier studies where EL is influential of producing an immediate increase testosterone production in intact rats. ${ }^{25,26}$ It promoted testosterone steroidogenesis at the rat testicular Leydig's cells by inhibiting aromatase transformation of testosterone to estrogen. ${ }^{27}$ No significant differences of testosterone level was detected when both groups treated with $15 \mathrm{mg} / \mathrm{kg}$ of EL was compared with each other (NDEL and HFDEL), this observation is consistent with former studies on phytoandrogenic effect of EL in rats and showed that El act as testosterone booster, ${ }^{24}$ and might be ascribed to the valuable effect of EL itself rather than to the differences in fat content of the diet, thus supplying further indication of the folk-use of EL as aphrodisiac and testosterone promoter. The effect of EL on somniferous tubules architecture demonstrated some optimistic protective findings. There were noticeable improvements observed in the HFDEL group compared to the severe deterioration in somniferous tubules of HFD group, indicating the testicular protective potential of EL. These deleterious effects of HFD have been abrogated with the administration of EL extract.

The mechanisms underlying this effect remain far less clear. A former study has proposed mechanisms that EL has the capability to activate the steroid 17 a monooxygenase to promote the metabolism of pregnenolone and 17 hydroxy pregnenolone to yield more dehydroepiandrosterone (DHEA), 4-androstenedione, and eventually to produce testosterone. ${ }^{26,23}$ Other investigators showed that the presence of the specific inhibitors for the cytochrome P450scc (CYP11a),17,20-lyase,17 $\beta$-hydroxy-steroid-dehydrogenase(CYP17a) 
and calcium ion channel such as aminoglutethimide, ketoconazole and the calcium ion channel blocker, nifedipine, significantly influenced the testosterone levels. ${ }^{27}$ -

EL root extract was outstanding and successful in the management of male infertility in. This is associated with the testosterone-enhancing effect of EL; that in turn, may activate spermatogenesis in testicles and improve the quality and quantity of sperms in ejaculate. ${ }^{29}$ In addition, the elevation in sperm concentration in sub-fertile men might also be assigned to the suppression of apoptosis of sperms in the presence of anti-sperm antibodies. ${ }^{30}$ In the present study the apoptosis of the spermatogenic cells was reduced in EL treated groups. Other studies have linked the positive effects of EL on male's fertility to the increased level testosterone as well as to its anti-oxidative features. ${ }^{31}$ Previous study has shown that antioxidant supplementation could improve male reproductive activities. ${ }^{32}$ However, other investigators have suggested that the eurypeptides (the prime of component the EL) may have played eminent role in liberating the bound-form of testosterone from sex hormone-binding globulin that might raise free form of sex hormone in the serum. ${ }^{33,34}$

\section{CONCLUSION}

The results of this indicate that Eurycoma longifolia root extract has a preventative effect against testicular injury which is induced by high fat diet. Further ultrastructural study is in progress to evaluate the cytomorphological changes in the testes of rats treated with HFD and the specific role of EL in protection.

\section{ACKNOWLEDGMENT}

The authors would like to thank International Islamic University Malaysia for funding this project under P RIGS grant number:18-030-0030.

\section{REFERENCES}

1. Han F, GuoY, Xu L, Hou N, Han F, Sun X. Induction of haemeoxygenase-1 directly improves endothelial function in isolated aortas from obese rats through the Ampk-Pi3k/Akt-Enos pathway. Cellular Physiology and Biochemistry. 2015;36(4):1480-90.

2. WHO. Obesity and overweight. February 2018.

3. Sohrabi M, Roushandeh AM, Alizadeh Z, Vahidinia A, Vahabian M, Hosseini $M$. Effect of a high fat diet on ovary morphology, in vitro development, in vitro fertilisation rate and oocyte quality in mice. Singapore Medical Journal. 2015;56:573-9.

4. Harper JW, Zisman TL. Interaction of obesity and inflammatory bowel disease. World Journal of Gastroenterology. 2016;22:7868-81.

5. Chambers TJ, Richard RA. The impact of obesity on male fertility. Hormones. 2015;14:563-68.

6. Fan $Y$, Liu $Y$, Xue K, Gu G, Fan W, Xu Y, et al. Diet-induced obesity in male C57BL/6 mice decreases fertility as a consequence of disrupted blood-testis barrier. PLoS ONE. 2015;10(4): e0120775.

7. YiX, Gao H, Chen D, Tang D, Huang W, LiT, et al. Effects of obesity and exercise on testicular leptin signal transduction and testosterone biosynthesis in male mice. American Journal of Physiology. 2017;312: R501-10.

8. Yang MU, Wen-Jie Y, Tai-Lang Y, Yang J. Curcumin ameliorates high-fat diet-induced spermatogenesis dysfunction. Molecular Medicine Reports. 2016;14:3588-94.

9. Tüfek NH, Altunkaynak ME, Altunkaynak BZ, Kaplan S. Effects of thymoquinone on testicular structure and sperm production in male obese rats. Systems biology in reproductive medicine. 2015;61(4):194-204

10. ALjuaid SM, Amin HA. Potential protective role of cinammon aquaous extract against hypercholesterolemia induced testicular damage in rats. Scholars Journal of Applied Medical Sciences. 2016;4(9E):3530-5.
11. Abdul Wahab N, Mokhtar NM, Halim WN, Das S. The effect of Eurycoma longifolia Jack on spermatogenesis in estrogen-treated rats. Clinics. 2010;65(1):93-8.

12. Faisal GG, Zakaria SM, Najmuldeen GF, Al-Ani IM. Antifungal activity of Eurycoma longifolia Jack (tongkat ali) root extract. Journal of International Dental and Medical Research. 2016;9:70-4.

13. Ang HH, NgaiTH, Tan TH. Effects of Eurycoma longifolia Jack on sexual qualities in middle aged male rats. Phytomedicine. 2003;10(6):590-3.

14. Bhat R, Karim AA. Tongkat ali (Eurycoma longifolia Jack): A review on its ethnobotany and pharmacological importance. Fitoterapia. 2010;81(7):669-79.

15. Talbott SM, Talbott JA, George A, Pugh M. Effect of Tongkat Ali on stress hormones and psychological mood state in moderately stressed subjects. J Int Soc Sports Nutr. 2013;10(1):28.

16. Al-Joufi FA. The effect of eurycoma Iongifolia on cardiovascular related parameter in rats fed high-fat diet. Ph.D. Thesis, International Islamic university malaysia 2016.

17. Mokhtar RH, Al-Joufi F, Saxena AK, Al-Ani IM, Talib NA, Ku-Zaifah N. The effects of Eurycoma longifolia on testosterone and blood pressure in high-fat-fed animal model. Journal of Applied Pharmaceutical Science. 2017;7:119-24.

18. Campos-Silva P, Furriel A, Costa WS, Sampaio FJ, Gregório BM. Metabolic and testicular effects of the long-term administration of different high-fat diets in adult rats. International Brazilian Journal of Urology. 2015;41(3):569-75.

19. Cui $X$, Long C, Tian J, Zhu J. Protective effects of Fluvastatin on reproductive function in obese male rats induced by high-fat diet through enhanced signaling of mTOR. Cell Physiology and Biochemistry. 2017;41:598-608.

20. Fernandez CD, Bellentani FF, Fernandes GSA, Perobelli JE, Favareto AP, Nascimento $A F$, et al. Diet-induced obesity in rats leads to a decrease in sperm motility. Reproductive Biology Endocrinology. 2011;9:32.

21. Macrini DJ, Bonamin LV, Teodorov EK, Thiago BC, Coelho CP. Overweight male juvenile rats exhibit decreases in sexual behavior and serum testosterone levels and an increase in TNF- $\alpha$ levels in adulthood. Psychology and Neuroscience. 2016;9(2):188-97.

22. Fan $W, X u Y$, Liu Y, Zhang Z, Lu L, Ding Z. Obesity or overweight, a chronic inflammatory status in male reproductive system, leads to mice and human subfertility. Frontiers in Physiology. 2017;8:1117.

23. Jia YF, Feng Q, Ge ZY, Guo Y, Zhou F, Zhang KS. Obesity impairs male fertility through long term effects on spermatogenesis. BMC Urology. 2018;18:42.

24. George A, Henkel R. Phytoandrogenic properties of Eurycoma longifolia as natural alternative to testosterone replacement therapy. Andrologia. 2014;46:708-21.

25. Tajul Ariff AS, Soelaiman IN, Pramanik J, Shuid AN. Effects of Eurycoma longifolia on testosterone level and bone structure in an aged or chidectomised rat model. Evid Based Complement Alternat Med. 2012;2012.

26. Low BS, Das PK, Chan KL. Standardized quassinoid rich Eurycoma longifolia extract improved spermatogenesis and fertility in male rats via the hypothalamicpituitarygonadal axis. J Ethnopharmacol. 2013b;145:706-14.

27. Rehman SU, Choe K, Yoo HH. Review on a traditional herbal medicine, Eurycoma longifolia Jack (Tongkat Ali): its traditional uses, chemistry, evidencebased pharmacology and toxicology. Molecules. 2016;21:331.

28. Tambi MI. Nutrients and botanicals for optimizing men's health. Examining the evidence for Eurycoma longifolia Jack, the Malaysian ginsing in men's health. Asian Journal of Andrology. 2009;11:37-8.

29. Thu HE, Mohamed IN, Hussain Z, Jayusman PA, Shuid AN. Eurycoma longifolia as a potential adoptogen of male sexual health: a systematic review on clinical studies. Chinese Journal of Natural Medicines. 2017;15(1):0071-80.

30. Pentikäinen V, Erkkilä K, Suomalainen L, Parvinen M, Dunkel L. Estradiol acts as a germ cell survival factor in the human testis in vitro. Journal of Clinical Endocrinology and Metabolism. 2000;85(5):2057-67.

31. Tambi MI. Eurycoma longifolia Jack: A potent adaptogen in the form of water soluble extract with the effect of maintaining men's health. Asian Journal of Andrology. 2006;8(Supp1):49-50.

32. Kefer JC, Agarwal A, Sabanegh E. Role of antioxidants in the treatment of male infertility. International Journal of Urology. 2009;16(5):449-57.

33. Exton MS, Krüger TH, Bursch N, Haake P, Knapp W, Schedlowski M, et al Endocrine response to masturbation-induced orgasm in healthy men following a 3-week sexual abstinence. World Journal of Urology. 2001;19(5):377-82.

34. Jiang M. Periodic changes in serum testosterone levels after ejaculation in men. Acta Physiologica Singapore. 2002;54(6):535-538. 